\title{
A Case of a Sudden Onset of Diplopia Revealing Third Oculomotor Palsy Due to Midbrain Hematoma
}

\author{
Sara Sabiry, Salma Bellakhdar, Hajar Khattab, Hicham El Otmani, Bouchra EL Moutawakil, Mohamed Abdoh Rafai
}

Hassan II University of Casablanca, Department of neurology and Clinical neurophysiology, IBN ROCHD University Hospital of Casablanca, Casablanca, Morocco

DOI: $10.36347 /$ sjmcr.2020.v08i07.014

| Received: 15.07.2020 | Accepted: 23.07.2020 | Published: 28.07.2020

*Corresponding author: Sara Sabiry

Abstract

Case Report

Spontaneous brainstem hemorrhage accounts for 5\%-10\% of intracerebral hemorrhage. Vascular diseases in the midbrain can cause oculomotor nerve palsy (ONP) often associated with other neurological signs. Isolated ONP due to hemorrhagic stroke restricted to the midbrain is considered to be rare. We report the case of a 54 years old women admitted with isolated third nerve palsy secondary to a midbrain hematoma.

Keywords: Brainstem hemorrhage, Midbrain hematoma, Oculomotor nerve palsy.

Copyright @ 2020: This is an open-access article distributed under the terms of the Creative Commons Attribution license which permits unrestricted use, distribution, and reproduction in any medium for non-commercial use (NonCommercial, or CC-BY-NC) provided the original author and source are credited.

\section{INTRODUCTION}

The third cranial nerve controls various movements of the eyeball, eyelid and sends parasympathetic fibers to the ciliary body and pupil [1]. Vascular diseases in the midbrain can cause oculomotor nerve palsy (ONP), often associated with conscious disturbance, hemiplegia, and other ocular symptoms. Usually, the causal lesion involves the pons or the thalamus. Isolated ONP due to hemorrhagic stroke restricted to the midbrain is considered to be rare. Few sporadic cases are reported in the literature. We report the case of a 54 years old women admitted with isolated third nerve palsy secondary to a midbrain hematoma.

\section{Case Report}

A 54 years old female, with a recent medical history of untreated hypertension and no other risk factors, was admitted to our hospital for the sudden onset of horizontal diplopia and left eye ptosis that she had been experiencing for 5 days, without headache, nausea, vomiting, weakness, or numbness. The symptoms were constant with no fluctuation.

The ocular motility examination revealed:

- On the left : ptosis, left pupillary dilation, loss of direct/indirect light reflexes, limitation in adduction, left downward and upward eye movement, limitation of convergence of the left eye,

- On the right: vertical gaze palsy of the right eye.
In conclusion, the examination concludes to a complete extrinsic and intrinsic left third cranial nerve palsy.

Visual acuity and intraocular pressure in both eyes were normal. His blood pressure was 220/100 $\mathrm{mmHg}$ on admission. The remaining results of her neurological and physical examinations were unremarkable.

Initial laboratory findings (coagulation time, full blood count, renal function, electrolytes, serum glucose) were normal.

Brain computed tomography (CT) on admission revealed a small spontaneously high-density lesion of the midbrain suggesting a midbrain hematoma. Cerebral Magnetic resonance imaging (MRI) revealed a small high-intensity in the midbrain on T1, T2-weighted and Fluid-attenuated inversion recovery (FLAIR) sequences (Figure 1 A,B,C) consistent with subacute midbrain hematoma associated to periventricular white matter hyperintensities. The T2* weighted gradientecho (GE) sequence (Figure 1D) showed a small signal loss in the midbrain associated with microbleeds mainly concentrated in basal ganglia, brainstem, and white matter of cerebellar hemispheres, compatibles with hemosiderin deposites from chronic bleeding in the context of hypertensive microangiopathy. 
We treated him conservatively with a monocular occlusion as the initial treatement for diplopia. Antihypertensive treatment was also started, by using IV Nicardipine initially then by switching to an oral association of calcium channel blockers and angiotensin converting enzyme inhibitors. Diplopia, ptosis and oculomotor function gradually improved over few months of follow-up.
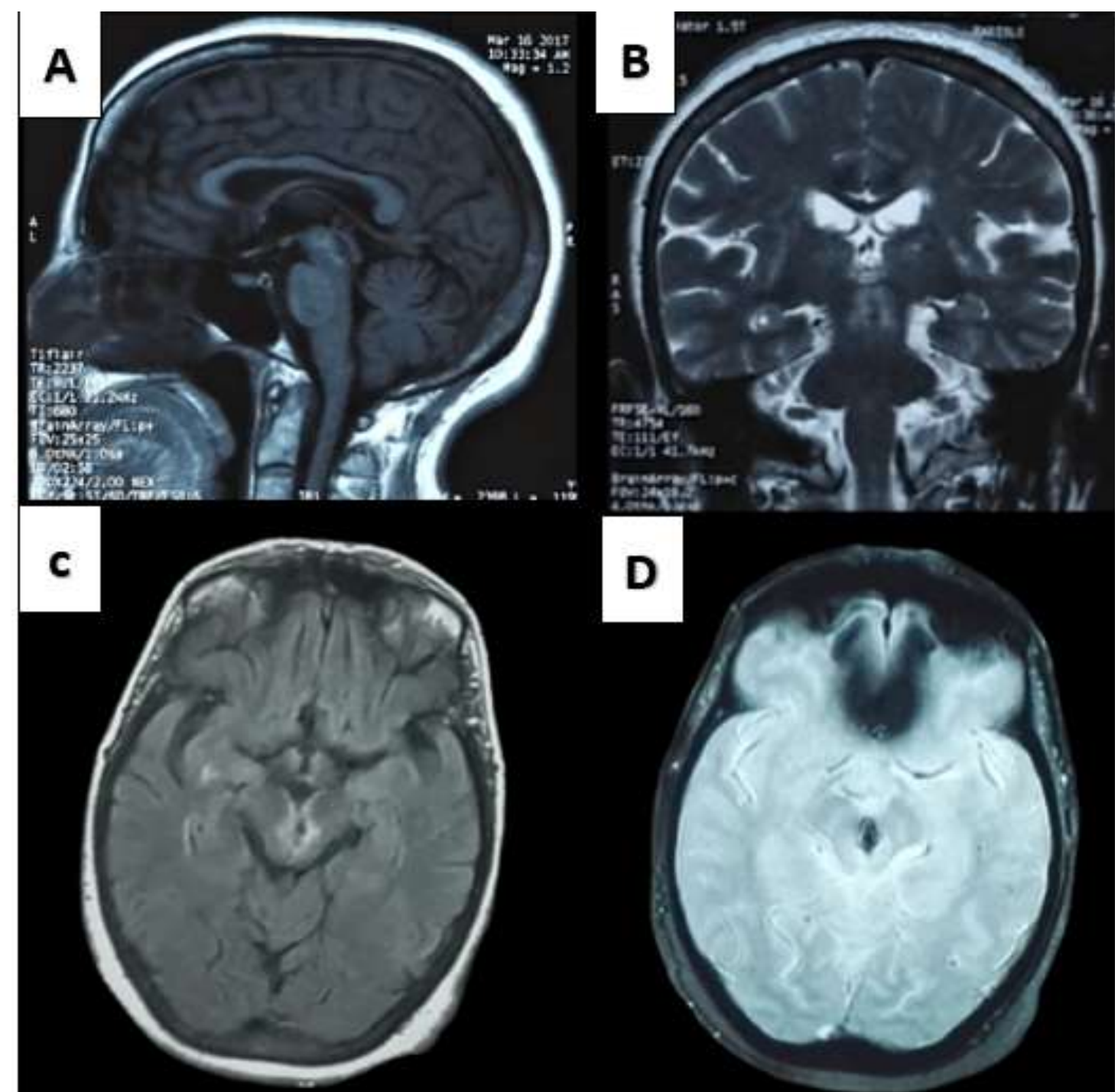

Fig-1: Brain MRI (A, B, C, D): Sagittal T1 and coronal T2-Weighted, axial FLAIR, and T2* weighted gradient-echo (GE) sequences demonstrated a small focal midbrain hematoma associated to periventricular white matter hyperintensities

\section{DiSCUSSION}

The third cranial nerve originates from the oculomotor nucleus, located within the centromedial part of the rostral midbrain of the brainstem, ventral to the cerebral aqueduct and dorsal to the medial longitudinal fasciculus. It emerges from the anterior aspect of the midbrain, passing inferiorly to the posterior cerebral artery and superiorly to the superior cerebellar artery.

The oculomotor nerve nucleus is composed of the lateral somatic cell column, the caudal central nucleus, and the medial cell column. The caudal central nucleus regulates the superior palpebral levator muscles bilaterally. The medial cell column regulates the contralateral superior rectus muscle. The lateral somatic cell column includes 3 nuclei (dorsal subnucleus, intermediate column, and ventral subnucleus) and regulates the inferior rectus, inferior oblique, and medial rectus muscles on the ipsilateral side [2]. With its parasympathetic fibers emerging from the EdingerWestphal nuclei, it innervates the iris constrictor and the annular portion of the ciliary muscle.

ONP generally leads to mydriasis, ptosis, adduction impairment and upward/downward gaze palsy in the ipsilateral affected eye. Furthermore our patient had, in addition to these classic findings, a vertical upward gaze palsy of the right eye. It is known that the fibers destined for the inferior rectus, inferior oblique, and medial rectus muscles supply the ipsilateral eye. When the palsy results from injury to the third nerve nucleus on one side, the patient may present 
with ptosis and supraduction weakness in the contralateral eye because of bilateral innervation of the superior palpebral levator muscles by the central caudal nucleus and due to the fibers' decussation of the superior rectus nucleus [3]. Vertical gaze palsy may also be caused by impairement of the ipsilateral rostral interstitial nucleus of the medial longitudinal fasciculus (riMLF) who is located in the rostral part of the oculomotor nerve nucleus in the diencephalon [2]. In our patient, the bilateral vertical gaze palsy can be explained by both nuclear and/or fascicular involvement.

Various disorders may cause ONP: infectious, traumatic, toxic, and vascular metabolic disorders. Stroke and particularly hemorrhage restricted to the midbrain is considered to be uncommon. Only a few sporadic cases of midbrain hematoma revealed by a sudden onset of diplopia with third oculomotor palsy have been reported. In our patient the midbrain hemorrhage was confirmed by brain CT and MRI.

In general, the most common causes of midbrain hemorrhage are vascular malformation, hypertension, and bleeding diathesis [3, 4].

Spontaneous brainstem hemorrhage accounts for $5 \%-10 \%$ of intracerebral hemorrhage, and secondary brainstem hemorrhage develops from cerebral hemorrhage with a frequency of $45 \%$ [5]. Most of the hematomas in brainstem originate from the branches of the basilar artery supplying the pons. Midbrain and medulla oblongata are practically less common sites of origin of brainstem hemorrhage [6].

Our patient had a sudden onset of symptoms in a context of high blood pressure $(220 / 100 \mathrm{mmHg})$. Brain imaging demonstrates a midbrain hematoma in the setting of hypertensive microangiopathy. The majority of spontaneous intracerebral hemorrhage is related to hypertensive small vessel disease [7]. In hypertensive patients, the most common site of brainstem hematoma is the pons. Midbrain localization is much rarer [8]. The location of the hematoma in deep area and associated neuroimaging markers can help the clinician to suggest the causality of hypertensive microangiopathy. In our patient, we observed a midbrain hematoma associated to periventricular white matter hyperintensities and deep microbleeds. All of this data is consistent with hemorrhagic midbrain stroke consecutive to hypertension.

Treatment options for diplopia include strabismus surgery, ocular occlusion, monovision optical correction, prism glasses, and chemodenervation by botulinum toxin $[9,3]$, associated with hypertensive management. We choose monocular occlusion for our patient. After few months, we observed a progressive remission of the ptosis and the diplopia. The hypertension was also controlled.

\section{CONCLUSION}

Six to ten percent of all parenchymatous brain hemorrhages are located in the brainstem, especially in the pons. Only few cases of focal midbrain hematoma revealed by a sudden onset of diplopia with third oculomotor palsy have been reported in the literature. This is an exceptional situation that must be considered because of the severity and therapeutic implications of the underlying disease.

Conflicting interest: The authors report no conflicts of interest

Acknowledgement: The authors state no additional individuals to acknowledge

\section{REFERENCES}

1. Yanovitch T, Buckley E. Diagnosis and management of third nerve palsy. Curr Opin Ophthalmol. 2007;18:373-8.

2. Ogawa K, Suzuki Y, Takahashi K, Kamei S, Ishikawa H. Clinical study of eleven patients with midbrain infarction-induced oculomotor nerve palsy. Journal of Stroke and Cerebrovascular Diseases. 2016 Jul 1;25(7):1631-8.

3. Lee CY, Chen HC, Lin HY, Kao LY. Oculomotor palsy as a single presenting sign of midbrain hemorrhage. Taiwan journal of ophthalmology. 2018 Apr;8(2):108-110.

4. Mizushima H, Seki T. Midbrain hemorrhage presenting with oculomotor nerve palsy: case report. Surgical neurology. 2002 Dec 1;58(6):41720.

5. Aguilar MI, Brott TG. Update in intracerebral hemorrhage. Neurohospitalist. 2011;1:148 - 59.

6. Raison JS, Bourbotte G, Baum TP. Primary brain stem hemorrhage: retrospective study of 25 cases. Revue Neurologique. 2008 Mar 11;164(3):225-32.

7. Pasi M, Marini S, Morotti A, Boulouis G, Xiong L, Charidimou A, Ayres AM, Lee MJ, Biffi A, Goldstein JN, Rosand J. Cerebellar hematoma location: implications for the underlying microangiopathy. Stroke. 2018 Jan;49(1):207-10.

8. Link MJ, Bartleson JD, Forbes G, Meyer FB. Spontaneous midbrain hemorrhage: report of seven new cases. Surgical neurology. 1993 Jan;39(1):58-65.

9. Sadagopan KA, Wasserman BN. Managing the patient with oculomotor nerve palsy. Curr Opin Ophthalmol. 2013;24:438- 47. 